\title{
O PET- Saúde na formação acadêmica em Odontologia: contribuições e perspectivas
}

Gabriela Crusoé Lopes Leite de Souza ${ }^{*}$, Maria Rita Lima Lopes ${ }^{*}$,Carlos Alberto Quintão Rodrigues $^{* *}$, Kênia Souto Moreira ${ }^{* * *}$, Michelle Pimenta Oliveira ${ }^{* * * *}$, Ariadna Janice Drumond Morais $^{* * * * *}$, Maisa Tavares de Souza Leite ${ }^{* * * * * *}$

* Acadêmicas do Curso de Graduação de Odontologia da Universidade Estadual de Montes Claros - Unimontes

** Mestre em Ciências da Saúde. Coordenador do Pró-Saúde/PET-Saúde e da Comissão de Residência Multiprofissional em Saúde - COREMU -da Universidade Estadual de Montes Claros - Unimontes

*** Mestranda em Cuidado Primário em Saúde. Enfermeira da Secretaria Municipal de Saúde de Montes Claros e preceptora do PET Saúde da Unimontes

**** $\quad$ Mestre em Cuidado Primário em Saúde. Cirurgiã-dentistada Secretaria Municipal de Saúde de Montes Claros e Preceptora do PET Saúde da Unimontes

Mestranda em Cuidados Primária em Saúde. Médica da Secretaria Municipal de Saúde de Montes Claros e preceptora do PET Saúde da Unimontes

Doutora em Ciências. Tutora do PET-Saúde da Unimontes

\section{RESUMO}

O Programa de Educação pelo Trabalho para a Saúde (PET-Saúde) oferece aos acadêmicos da graduação a inserção nos serviços de saúde, permitindo que os mesmos vivencie a realidade desses serviços. $\mathrm{O}$ PET-Saúde visa desenvolver primordialmente, habilidades necessárias para uma atuação em equipes multiprofissionais. Utiliza-se a metodologia de observação, vivência e pesquisa para a construção do conhecimento. A articulação entre ensinoserviço-comunidade proporciona uma aprendizagem única aos acadêmicos, aos profissionais da rede de atenção à saúde e a própria comunidade. Assim, o PET-Saúde visa à formação de profissionais de saúde com elevada qualificação técnica, científica e acadêmica. O objetivo do presente trabalho é relatar as experiências vividas pelos acadêmicos de odontologia no desenvolvimento de ações de ensino, pesquisa e extensão realizadas pelo PET-Saúde, e suas perspectivas na formação profissional. $\mathrm{O}$ curso de graduação em Odontologia tem sofrido varias mudanças desde sua criação, sendo o
PET-Saúde um excelente meio de aplicar essa nova visão que se tem dado a essa graduação, sendo um programa que leva o acadêmico a vivências extramuros, propiciando um maiorconhecimento, por parte dos alunos das estruturas dos serviços públicos de saúde. As atividades de ensino, pesquisa e extensão vividas no PET-Saúde contribuem para um crescimento integrado dos acadêmicos, que aprendem fundamentos essenciais para sua vida profissional.

Descritores: Ensino; Atenção primária à saúde; Odontologia.

\section{INTRODUÇÃO}

O Programa de Educação pelo Trabalho para a Saúde (PET-Saúde) foi instituído pela Portaria Interministerial MS/MECn ${ }^{\circ}$ 421, de 03 de março de 2010, sendo destinado a fomentar grupos de aprendizagem tutorial em áreas estratégicas para o Sistema Único de Saúde (SUS) ${ }^{1}$.

Oferece aos acadêmicos da graduação a inserção nos serviços públicos de sa- 
úde, por meio de estágios e vivências proporcionados pelas instituições de ensino superior em parceria com as Secretarias Municipais de Saúde, disponibilizando bolsas de educação pelo trabalho e incentivo à produção de conhecimento científico direcionado à qualificação dos serviços públicos de saúde ${ }^{2}$.

A articulação entre ensino-serviçocomunidade proporciona simultaneamente oportunidade de uma aprendizagem significativa aos graduandos, inclusão de profissionais da rede de atenção à saúde no processo de ensino-aprendizagem e produção de conhecimento científico, e melhoria nos determinantes do processo saúde-doença nas comunidades assistidas ${ }^{3}$. Nessa perspectiva, a interação ensino e serviço traz possibilidades e desafios aos sujeitos envolvidos, contribuindo para re-significação do papel dos profissionais, co-protagonistas na construção do sistema de saúde ${ }^{4}$.

Essa articulação favorece que a academia integre, durante todo o processo de ensino-aprendizagem, a orientação teórica com as práticas de atenção nos serviços públicos de saúde, em sintonia com as reais necessidades dos usuários do SUS $^{5}$.

Assim, o PET-Saúde visa à formação de profissionais de saúde de elevada qualificação técnica, científica e acadêmica, pautada pelo espírito crítico, pela cidadania e pela função social da educação superior, orientada pelo princípio da indissociabilidade entre ensino, pesquisa e extensão, levando o acadêmico a experiências extramuros, aprimorando seu olhar, fazendo-o a entender o processo de trabalho em equipe, reconhecendo a realidade social, além de desenvolver e participar de estudos científicos e outras formas de produção de conhecimento que possam qualificar a prática profissional $^{2,4,6}$.
No município de Montes Claros/MG é desenvolvido o Pró-Saúde/PET-Saúde Redes de Atenção à Saúde, uma parceria entre a Universidade Estadual de Montes Claros - Unimontes - e a Secretaria Municipal de Saúde de Montes Claros. Esse programa conta com a participação dealunos dos cursos de odontologia, medicina, enfermagem, ciências biológicas, educação física e serviço social.

Segundo Mendes $(2011)^{7}$ as redes de atenção à saúde são consideradas como uma nova forma de organizar o sistema de atenção à saúde em sistemas integrados que permitam responder, com efetividade, eficiência, segurança, qualidade e equidade, às condições de saúde da população. Sendo as mesmas organizações poliárquicas de um conjunto de serviços de saúde quepermitem ofertar uma atenção contínuae integral a determinada população,coordenada pela atenção primária à saúde (APS) ${ }^{8}$.

Devido a esse sistema de serviço de saúde ser coordenado pela APS, o PETSaúde da Unimontes desenvolve a maioria de suas ações voltadas para esse nível de atenção, sendo a Estratégia Saúde da Família (ESF) um excelente campo de inserção para os acadêmicos.

Diante do amplo cenário ofertado pela Rede de Atenção à Saúde, os acadêmi$\cos$ são incentivados a realizar diversas ações não só no campo clínico, mas também no campo comunitário. Portanto o objetivo desse trabalho é relatar as experiências vividas pelos acadêmicos de odontologia no desenvolvimento de ações de ensino, pesquisa e extensão realizadas pelo PETSaúde, e suas as perspectivas na formação profissional.

\section{METODOLOGIA}

O trabalho em questão é um relato 
de experiência de caráter descritivo de acadêmicos do curso de odontologia Unimontes inseridos no Pró-Saúde/PET-Saúde Redes de Atenção à Saúde. As atividades descritas foram desenvolvidas em dois cenários da Atenção Primária à Saúde, envolvendo duas equipes da Estratégia Saúde da Família da Rede de Atenção à Saúde do município de Montes Claros - MG. As atividades acadêmicas desenvolvidas no Programa compreenderam estratégias e metodologias ativas de ensino-aprendizagem.

\subsection{Descrição da experiência}

Inicialmente foi realizada uma capacitação para os acadêmicos, em que os preceptores discorriam através de aulas e distribuição de material didático sobre vários temas pertinentes a inserção no território. Assuntos como diagnostico local, teritorialização, visita domiciliar, ferramentas de família como o genograma entre outros. Assuntos estes que não eram de domínio da maioria dos acadêmicos.

Isso foi essencial para o aprofundamento sobre o que é a ESF, como ela é organizada e suas principais ações. Desta forma, no momento em que realmente houve a inserção no território a experiência foi mais exitosa haja vista que já se tinha um conhecimento prévio do que era a ESF e do processo de trabalho desta.

Em seguida foi feito diagnóstico local com a territorialização da área de abrangência das duas ESF vinculadas ao PET-Saúde, e o diagnostico de saúde da população, identificando as doenças individuais e os problemas de saúde coletiva mais prevalentes. Esse momento foi de reflexão e a partir dos problemas encontrados foram planejadas ações preventivas, promocionais e curativas.

Os Centros Municipais de Educação Infantil foram locais escolhidos para o de- senvolvimento de atividades educativas, locais esses que se mostraram excelentes multiplicadores de conhecimento. Ações educativas por meio de palestras, teatros, vídeos foram realizadas com sucesso, sendo abordados temas como alimentação saudável, higiene oral, cuidados com a dengue entre outros.

Ações mais direcionadas para a odontologia também foram realizadas, como a escovação supervisionada, levantamentos de necessidades bucais e o tratamento restaurador atraumático-ART, sendo essas também vinculadas ao programa Saúde na escola. Essas ações contribuíram em grande escala no aperfeiçoamento dos acadêmicos de odontologia, uma vez que os mesmos foram levados a praticas odontológicas fora dos muros da universidade.

Foi desenvolvido na semana da criança o "Cinema Divertido" na sala de eventos da paróquia do bairro. $\mathrm{O}$ cinema foi feito com a escolha de filmes interativos e aproveitou-se o momento para o acompanhamento infantil, fez-se pesagens, mediuse o comprimento e promoveu saúde geral, através de diversas ações dinâmicas.

Um dos principais objetivos do PET-Saúde Unimontes foi possibilitar uma atenção integral às crianças da área de abrangência e influencia da ESF. Assim diversas ações foram desenvolvidas nesse contexto nas sedes das ESF's e na comunidade.

Um exemplo disso são as Consultas de Crescimento e Desenvolvimento Infantil (CD). São consultas multiprofissionais com a inserção inédita do profissional cirurgiãodentista. Essas foram agendadas pelos agentes comunitários, através da visita domiciliar e/ou puerperal e pela livre demanda da unidade. As consultas multiprofissionais ocorreram sob a preceptoria de profissional enfermeiro, médico e cirurgião-dentista e 
acompanhados de acadêmicos de cursos de graduação em enfermagem, medicina, odontologia e serviço social, integrantes do PET-Saúde. A consulta de Crescimento e Desenvolvimento das crianças realizada de forma multiprofissional favoreceu de modo geral a troca de experiências dentro da equipe de saúde, melhorou a qualidade de vida das crianças adscritas, promoveu a saúde; possibilitou o fortalecimento do trabalho multiprofissional, garantiu uma adesão precoce e atraumática das crianças ao tratamento odontológico, além de atingir os princípios básicos do Sistema Único de Saúde.

Outro exemplo da interdisciplinaridade proposta pelo PET-Saúde foiuma atividade que marcou a comunidade, os profissionais e os acadêmicos, momento único e de grande contribuição para a formação profissional.

A ação foi realizada na Unidade Básica de Saúde, sendo que cada micro área foi atendida em um sábado do mês. O público alvo foram as crianças de 2 a 5 anos acompanhadas pelos pais. Primeiramente foi feito um teatro intitulado "A Estrelinha Azul" que falava sobre os cuidados com a higiene, em seguida foi realizada uma palestra sobre alimentação saudável, hábitos de higiene corporal e Saúde bucal. Após esse momento de educação em saúde, na sala de espera foi exibido um vídeo educativo, enquanto se aguardava o atendimento multiprofissional e os pais receberam informações sobre o que era o Centro de Referencia de Assistência Social- CRAS e os seus benefícios para a comunidade.

As crianças foram pesadas e medidas, em seguida passaram por uma consulta médica, odontológica e de enfermagem, em que esses profissionais atenderam juntos, um contribuindo para a construção do co- nhecimento do outro. Foi feito o levantamento de necessidades bucais e as crianças que tinham mais demanda foram atendidas imediatamente após a consulta multiprofissional e as demais agendadas para outra data. Ao final da atividade foi servida uma deliciosa salada de fruta para todos.

À exemplo de atividades realizadas na comunidade foi um evento de Natal em que a ESF em parceria com o PET levaram as crianças de alguns Centros Municipais de Educação para o evento "Natal no aeroporto". Nessa oportunidade, as crianças foram levadas ao aeroclube da cidade de Montes Claros para presenciarem a descida do Papai Noel Saudável no seu avião. Foram distribuídos presentes e desenvolvidas ações saudáveis para as crianças (teatro, brincadeiras, lanches) com o objetivo de fazer a alegria da criançada e levar conhecimento aos pequeninos, além de possibilitar um dinamismo de atividades e aguçar a fantasia das crianças pela presença do papai Noel.

O PET-Saúde tem se mostrado um ótimo meio para a disseminação dos princípios do modelo de Produção Social da Saúde, de forma que os acadêmicos são incentivados a todo momento a desenvolverem ações preventivas, promocionais e curativas direcionadas a todos os ciclos de vida. Grupos operativos, com hipertensos, diabéticos, idosos, gestantes e grupo de planejamento familiar foram também meios de disseminar conhecimento e aperfeiçoar a prática dos acadêmicos na ESF.

Como uma das diretrizes do PETSaúde é a realização da pesquisa seguindo linhas de conhecimento na área da Atenção Primária à Saúde, o PET-Saúde Unimontes elaborou sete projetos de pesquisa aprovados pelo comitê de ética que se encontra em execução, sendo eles: 
- Transtornos mentais menores: Prevalência nas mulheres em uma equipe de Saúde da Família;

- A saúde na perspectiva de indivíduos do sexo masculino não atendidos na Atenção Primária;

- Avaliação dos prontuários de hipertensos segundo o escore de framingham;

- Diagnóstico e análise da violência contra a mulher no município de montes claros - MG: pesquisa-ação;

- Prevenção da hipertensão arterial em crianças nos serviços de atenção primária à saúde;

- Análise da relação entre mudança de hábitos de vida e melhora do risco cardiovascular em adultos hipertensos;

- Avaliação do impacto de um programa de promoção de saúde para redução da ocorrência de cárie em pré-escolares.

Todos os projetos estão sendo desenvolvidospelos acadêmicos com orientação dos preceptores e tutorado PET Saúde Unimontes, e já possuem resultados parciais, resultados esses que já foram apresentados em congressos científicos.

\section{DISCUSSÃO}

O curso de graduação em Odontologia tem sofrido varias mudanças desde a sua criação, sempre sendo influenciado pelo modelo de saúde operante ${ }^{6,9}$. Vale ressaltar que algumas deficiências na formação universitária são constatadas pela dificuldade de alguns profissionais no redirecionamento das ações enfocando a família e a promoção da saúde, dentro da necessidade de atuação integral da $\mathrm{ESF}^{10}$.

As Diretrizes Curriculares Nacionais (DCN) aprovadas em 2002 para os cursos de odontologia, propostas pela Lei de Diretrizes e Bases da Educação(LDB) garante uma integração efetivaentre o ensino e os serviços ${ }^{9}$. Isso tem contribuído para a mudança nos currículos dessa graduação, na qual o aluno é incentivado a ser crítico, capaz de aprender aaprender, de trabalhar em equipe, de levar em contaa realidade social $^{6}$.Propõem um cirurgião-dentista com perfilgeneralista, com sólida formação técnico-científica,humanística e ética, orientada para a promoção de saúde ${ }^{9}$.

O PET-Saúde tem se mostrado um excelente meio de aplicar essa nova visão que se tem dado aos cursos de odontologia, sendo um programa que leva o acadêmico a vivências extramuros, propiciando um maiorconhecimento, por parte dos alunos das estruturas dos serviços públicos de saú$\mathrm{de}^{3,11}$.

Gonçalves et al. (2011) afirmam que o PET-Saúde propicia aos alunos de odontologia uma participaçãono atendimento à população, compreensãodas políticas de saúde bucal e do papel do cirurgiãodentista,conhecimento das bases epidemiológicasdo método clínico e de suas aplicações práticas nos

programas de saúde bucal.

Um dos eixos que norteia o PETSaúde é a interdisciplinaridade, isso talvez seja um dos maiores ganhos desse programa, poder compartilhar experiências, conhecimentos e realmente aplicar a visão holística e integrada do ser humano. Para Sales et al. (2011) ${ }^{12}$ a interdisciplinaridade implica uma consciênciados limites e das potencialidades de cada campo desaber para que possa haver uma abertura em direçãode um fazer coletivo.Sendo o objetivo das ações interdisciplinares no âmbito da atenção básica contemplar o tênue equilíbrio entre as demandas de saúdeda população e 
as possibilidades do Sistema de Saúde deatendê-las ${ }^{13}$.

O processo ensino-pesquisaextensão vivenciado pelos acadêmicos do PET-Saúde amplia ainserção destes no contexto social. Para Rodrigues et al. (2011) isso é importanteuma vez que, a reforma do setor saúde tornaa capacitação de recursos humanos um desafiomediante a transitoriedade do saber e das mudançasno mundo do trabalho.

O PET-Saúde possibilita ao discente conhecer melhor a dinâmica do trabalho em equipe,entendendo a interdependência positiva que existeentre os diferentes profissionais, isso se dá devido ao mesmo ser composto por uma equipe multi e interdiscipli$\operatorname{nar}^{12}$

Rodrigues et al. (2012) ${ }^{13}$ afirmam que o PET-Saúde prenuncia ser uma grande promessapara o aprimoramento da Atenção Básica, visto que traz propostasinovadoras para a ESF, fundamentadas nos princípios e diretrizes do SUS, além de possibilitar a construção de uma novaalternativa de educação e extensão, quebrando o modelotradicional de ensino ${ }^{4}$.

\section{CONCLUSÃO}

O PET- Saúde em toda a sua essência é uma projeto que tem marcado a vida de varias pessoas, ele proporciona a troca mutua entre profissionais do serviço, professores, acadêmicos e comunidade. As atividades de ensino, pesquisa e extensão contribuem para um crescimento integrado dos acadêmicos de odontologia, que aprendem fundamentos essenciais para sua vida profissional, pautado nos novos rumos que a odontologia tem seguido, não tendo mais seu enfoque somente no ambiente clínico, mas também na visão de serem mais preparados para atuar na comunidade, sobretudo em serviços de saúde.
De uma forma geral, o PET permite perceber que as ações focadas para as crianças tem um impacto positivo e a longo prazo na melhoria da qualidade de vida daqueles que serão os futuros adultos e corpo técnico das ações, além de possibilitar aos integrantes através das ações que são desenvolvidas uma formação generalista e humanizada enquanto profissionais da saúde, capacitando a agir dentro das diversas nuances e desafios do serviço público.

A interdisciplinaridade eleva o PET a um estado de excelência, criando a oportunidade de construção do conhecimento sob diversas visões não só humanas, mas também profissionais.

\section{REFERENCIAS}

1. Brasil. Ministério da Saúde. Ministério da Educação. Portaria Interministerial $\mathrm{n}^{\circ}$ 422, de 3 de março de 2010. Institui o Programa de Educação pelo Trabalho para a Saúde (PET Saúde) e dá outras providências.Diário Oficial da União. Brasília, 5 de março de 2010. Seção 1, p. 53.

2. Brasil. Ministério da Saúde. Ministério da Educação. Portaria Interministerial $\mathrm{n}^{\mathrm{o}} 1.802$, de 26 de agosto de 2008. Institui o Programa de Educação pelo Trabalhador para a Saúde - PETSaúde. Diário Oficial da União. Brasília, 27 ago. 2008; Seção 1, p.27.

3. Assega ML, Lopes Junior LC, Santos EV, Antoniass RS, PadulaMGC, Pirolo SM. A interdisciplinaridade vivenciada no PET-Saúde. Revista Ciência \& Saúde, Porto Alegre,2010, v. 3, n. 1, p. 2933.Acessado em $<$ http://revistaseletronicas.pucrs.br/ojs/i ndex.php/faenfi/article/viewFile/7027/5 915>.

4. Rodrigues CAQ, Costa SM, LeiteMTS, Mendes DC, Rodrigues Neto JF. A vi- 
vência dos estudantes deodontologia nas atividades de ensinopesquisaextensão do PET-Saúde. Revista da ABENO, 2011, v.11, n.2, p: 45-50.

5. Brasil. Ministério da Educação. Ministério da Saúde. Programa Nacional de Reorientação da Formação Profissional em Saúde - Pró-Saúde. Brasília; 2007.

6. Gonçalves CM, Santos KT, Carvalho RB. O PET-Saúde como instrumento de reorientação do ensino emOdontologia: a experiência daUniversidade Federal do Espírito Santo. Revista da ABENO, 2011, v. 11, n.2, p:27-33.

7. Mendes, EV. Revisão bibliográfica sobre redes de atenção à saúde. Belo Horizonte, Secretaria deEstado de Saúde de Minas Gerais, 2007.

8. Mendes, EV. As redes de atenção à saúde.Brasília: Organização PanAmericana da Saúde, 2011. Acessado em

$<$ http://www.saude.sp.gov.br/resources /ses/perfil/gestor/documentos-deplanejamento-em-saude/elaboracao-doplano-estadual-de-saude-2010-

2015/textos-de-

apoios/redes_de_atencao_mendes_2.pdf>

9. Brasil. Conselho Nacional de Educação. Diretrizes Curriculares Nacionais do Curso de Graduação em Odontologia. Resolução no CNE/CES 3/2002. Diário Oficial da União, Brasília, 4 mar. 2002. Seção 1, p. 10.

10. Moretti-Pires RO, Bueno SMV. Freire e formação para o Sistema Único de Saúde: o enfermeiro, o médico e o odontólogo. Acta Paul Enferm. 2009, v. 22, n. 4, p:439-44. Acessado em $<$ http://www.scielo.br/pdf/ape/v22n4/a $15 \mathrm{v} 22 \mathrm{n} 4 . \mathrm{pdf}>$

11. Moimaz SAS, Saliba NA, Garbin CAS, Zina LG. Atividades extramuros na ótica de egressos do Curso de Graduação em Odontologia. Revista da ABENO, 2008, v. 8, n.1, p: 23-9.

12. Sales KNA, Paula FA, Ribeiro M, Ribeiro LCC, Canuto SM. PET-Saúde: formando discentes multiplicadores relato de experiência. Revista da ABENO, 2011, v.11, n. 2, p:51-6.

13. Rodrigues AAAO, Juliano IA, Melo MLC, Beck CLC, Prestes FC. Processode Interação Ensino, Serviço eComunidade: a Experiência de um PETSaúde. Revista Brasileira de Educação Médica, 2012, v. 36, n. 1, p: $184-192$. Acessado em $<$ http://www.scielo.br/pdf/rbem/v36n1s 2/a27v36n1s2.pdf $>$

\section{ABSTRACT \\ The PET-Health in academic education in dentistry: contributions and prospects} The Education Program at Work for Health ( PET - Health ) offers academic graduation insertion in health services, allowing them to experience the reality of these services . The PET - Health aims primarily to develop necessary for a performance in multidisciplinary teams skills. Uses the methodology of observation, experience and research to build knowledge . The relationship between school-community-serviceprovides a unique to academics, professionals of health care and community network. Thus, PET - Health aims to train health care professionals with high technical, scientific and academic qualification. The objective of this study is to report the experiences of the dental students in actions of teaching, research and outreach conducted by PET Health, and its prospects in vocational training. The undergraduate course in dentistry has undergone several changes since its inception, the PET - Health is an excellent way to apply this new vision that has been Revista da ABENO • 14(1): 73-80, 2014 
given to this degree, one academic program leading to extramural experiences, providing greater knowledge, by students of structures of public health services. The activities of teaching, research and extension lived in PET - Health contribute to an integrated academics who learn essential foundations for your professional life growth.

Descriptors: Education, Primary Health Care; Dentistry. 